\title{
Miocardiopatia Diabética
}

revisão

KATASHI ОКОSHI

JuLliano F. CAMPOS GUIMARÃES Bruno PaUlino DI MUZIO

ANA ANGÉLICA H. FERNANDES Marina Politi OKoshi

Faculdade de Medicina de Botucatu, UNESP, SP.

Recebido em 18/11/06 Aceito em 23/1 1/06

\section{RESUMO}

A miocardiopatia diabética é uma doença do músculo cardíaco causada pelo diabetes mellitus e não relacionada às patologias vascular e valvular ou à hipertensão arterial sistêmica. Observações experimentais e clínicas têm demonstrado hipertrofia, necrose, apoptose e aumento do tecido intersticial miocárdico. Acredita-se que a miocardiopatia diabética seja decorrente de anormalidades metabólicas como hiperlipidemia, hiperinsulinemia e hiperglicemia, e de alterações do metabolismo cardíaco. Tais alterações podem causar aumento do estresse oxidativo, fibrose intersticial, perda celular e comprometimento do trânsito intracelular de íons e da homeostase do cálcio. Clinicamente, é possível a detecção de disfunção diastólica assintomática na fase inicial. No momento em que surgem os sinais e sintomas de insuficiência cardíaca, observamos disfunção diastólica isolada, sendo que o comprometimento da função sistólica, habitualmente, é tardio. $\mathrm{O}$ tratamento da miocardiopatia diabética com insuficiência cardíaca não difere das miocardiopatias de outras etiologias e deve seguir as diretrizes de acordo com o comprometimento da função ventricular, se diastólica isolada ou diastólica e sistólica. (Arq Bras Endocrinol Metab 2007;51/2:160-167)

Descritores: Miocardiopatia; Diabetes mellitus; Insuficiência cardíaca; Fisiopatologia; Tratamento

\section{ABSTRACT}

\section{Diabetic Cardiomyopathy.}

Diabetic cardiomyopathy is a myocardial disease caused by diabetes mellitus unrelated to vascular and valvular pathology or systemic arterial hypertension. Clinical and experimental studies have shown that diabetes mellitus causes myocardial hypertrophy, necrosis, and apoptosis, and increases interstitial tissue. The pathophysiology of diabetic cardiomyopathy is incompletely understood. It appears that metabolic perturbations such as hyperlipidemia, hyperinsulinemia, hyperglycemia, and changes in cardiac metabolism are involved in cellular consequences leading to increased oxidative stress, interstitial fibrosis, myocyte death, and altered intracellular ions transient and calcium homeostasis. Clinically, an early detection of asymptomatic diastolic dysfunction is possible. When patients develop signals and symptoms of heart failure, isolated diastolic dysfunction is usually detected. Systolic dysfunction is a late finding. Treatment of heart failure due to diabetic cardiomyopathy is not different from myocardiopathies of other etiologies and must follow the guidelines according to ventricular function, whether diastolic or diastolic and systolic impairment.

(Arq Bras Endocrinol Metab 2007;51/2:160-167)

Keywords: Cardiomyopathy; Diabetes mellitus; Heart failure; Pathophysiology; Treatment 
$\mathrm{O}$ CONTÍNUO AUMENTO DA PREVALÊNCIA e incidência de diabetes mellitus (DM) nas últimas décadas tem alertado a atenção médica para a necessidade de mudança de hábitos de vida da população com o objetivo de reduzir os fatores de risco para DM como obesidade, alimentação inadequada e inatividade física e, conseqüentemente, reduzir a ocorrência de complicações cardiovasculares.

Estudo com dados da Organização Mundial de Saúde estimou a prevalência de DM em 2,8\% na população mundial em 2000, o equivalente a 171 milhões de pessoas. Projetou, ainda, para 2030 a prevalência de $4,4 \%$ na população mundial, representando cerca de 366 milhões de pessoas acometidas pela doença (1). No Brasil, a prevalência observada de DM em 9 capitais entre 1986 e 1988, na faixa etária de 30-69 anos, foi de 7,6\% (2). Estudo recente mostrou, na mesma faixa etária, prevalência ainda maior $(12,1 \%)$ na população urbana de Ribeirão Preto, SP (3).

Diabetes mellitus é um distúrbio que consiste na resposta secretória defeituosa ou deficiente de insulina, manifestando-se pela utilização inadequada de glicose pelos tecidos com conseqüente hiperglicemia. Os distúrbios do metabolismo da glicose podem causar complicações que envolvem doenças cardiovasculares, incluindo hipertensão arterial sistêmica, doença arterial coronariana e insuficiência cardíaca, sendo que 75\% dos pacientes diabéticos morrem por algum evento cardiovascular (4-6).

Nas últimas décadas, e principalmente nos últimos anos, tem surgido grande número de evidências de que pacientes diabéticos sofrem também de uma forma de doença miocárdica não relacionada à doença arterial coronariana ou à hipertensão arterial sistêmica, denominada cardiomiopatia ou miocardiopatia diabética. Dentre os diversos problemas cardíacos que surgem em decorrência do DM, a miocardiopatia diabética tem sido reconhecida como uma doença cardíaca específica, que ocorre em aproximadamente 30\% dos pacientes diabéticos tipo $1(7,8)$.

\section{OBSERVAÇÕES EPIDEMIOLÓGICAS E CLÍNICAS}

Rubler e cols. (1972) foram os primeiros a introduzir o termo miocardiopatia diabética após o estudo postmortem de quatro pacientes diabéticos que tiveram insuficiência cardíaca congestiva na ausência ou evidência de doença arterial coronariana, valvulopatia, doença congênita, hipertensão arterial ou alcoolismo (7).

Levantamentos epidemiológicos realizados há mais de 30 anos já mostravam que homens diabéticos têm mais que o dobro de freqüência de insuficiência cardíaca (IC) do que os não-diabéticos, enquanto que as mulheres têm quintuplicado o risco para IC. O risco excessivo persiste independentemente de ajustes para idade, hipertensão arterial, obesidade, hipercolesterolemia ou doença arterial coronariana (9). Mais recentemente, nos grandes ensaios clínicos que avaliaram os inibidores da enzima conversora da angiotensina no tratamento da IC, como o SOLVD, ATLAS e V-HeFT II, foram observadas altas prevalências de DM, respectivamente 26\%, 19\% e 20\%, enquanto que a prevalência observada de DM na comunidade foi de 4 a 6\% (10-12).

De acordo com Rossen, pode existir correlação entre miocardiopatia diabética e microangiopatia, tendo em vista as semelhanças entre as anormalidades na função microvascular coronariana observadas no diabetes e na cardiomiopatia dilatada idiopática (13). Em cerca de $72 \%$ dos pacientes diabéticos normotensos foi observada doença evidente de pequenos vasos, enquanto que em pessoas não-diabéticas esse achado foi somente de $12 \%$ (14). Além disso, anormalidades da reserva de fluxo coronariano têm sido consistentemente demonstradas em pacientes diabéticos sem doença arterial coronariana epicárdica. Fibrose perivascular e intersticial e hipertrofia miocárdica foram também achados freqüentes em diabéticos $(15,16)$. No estudo UKPDS (United Kingdom Prospective Diabetes Study), os eventos cardiovasculares fatais foram $70 \%$ mais freqüentes que as complicações microvasculares, após 9 anos de seguimento $(17,18)$. Embora os doentes com DM tenham maior prevalência de dislipidemia, hipertensão arterial e obesidade, esses fatores isoladamente não justificam o aumento da mortalidade, sendo o DM aceito hoje como um importante fator de risco independente para o desenvolvimento de falência cardíaca.

Estudos em pacientes diabéticos sem IC mostraram comprometimento miocárdico de pequena monta em cerca de $20-40 \%$ dos doentes. Os pacientes diabéticos apresentavam elevação da pressão diastólica final do ventrículo esquerdo (VE) e redução do débito cardíaco quando confrontados com pacientes não-diabéticos. Pacientes com DM e que foram acometidos por infarto agudo do miocárdio apresentam aumento da prevalência de IC e pior evolução que os não-diabéticos, tendo disfunção diastólica mais severa quando comparados a indivíduos de grau semelhante de coronariopatia (19). Estudos ecocardiográficos em portadores de DM sem IC têm relatado hipertrofia ventricular esquerda e, sistematicamente, comprometimento da função diastólica do VE, principalmente alteração do relaxamento ven- 
tricular, mesmo na ausência de hipertrofia miocárdica $(20,21)$. Com a utilização de técnicas ecocardiográficas mais sofisticadas, foi demonstrada também disfunção sistólica do VE em pacientes diabéticos com fração de ejeção normal (22). Portanto, em indivíduos com DM e sem IC a disfunção diastólica do VE é um achado relativamente comum. No momento em que ocorrem manifestações clínicas de IC, o comprometimento diastólico pode ser observado praticamente em todos os pacientes. Posteriormente, com a progressão da doença, haverá também disfunção sistólica do VE.

\section{OBSERVAÇÕES EXPERIMENTAIS}

Vários modelos de experimentação animal são utilizados para o estudo da miocardiopatia diabética. $\mathrm{Na}$ indução de DM considerado do tipo l, a administração de estreptozotocina ou aloxana causa destruição de parte das células beta pancreáticas com conseqüentes hipoinsulinemia, hiperglicemia e manifestação de características observadas no diabetes tipo 1 como hiperfagia, polidipsia e perda de peso corporal. Nesses modelos, foram observados perda de proteínas contráteis, perda de miócitos e alterações na SERCA-2a com conseqüente diminuição do seqüestro de cálcio no retículo sarcoplasmático e sobrecarga intracelular de cálcio (23). Além disso, em nosso laboratório observamos aumento dos lípides séricos e aumento da atividade do estresse oxidativo (24). Provavelmente em decorrência da hipoinsulinemia, a hipertrofia miocárdica não tem sido habitualmente observada nesse modelo (23).

Para o modelo experimental de DM tipo 2, geralmente são utilizados ratos Zucker e camundongos geneticamente modificados $\mathrm{ob}^{-} / \mathrm{ob}^{-}$(deficiência de leptina) e $\mathrm{db}^{-} / \mathrm{db}^{-}$(deficiência de receptores de leptina). Nesses modelos, os animais desenvolvem obesidade, resistência à insulina com hiperinsulinemia e hiperglicemia. Hipertrofia miocárdica é a anormalidade cardíaca mais comumente observada (23).

Independentemente dos modelos experimentais, os animais diabéticos apresentam comprometimento da função diastólica do VE. Em relação à função sistólica do VE, a literatura fornece inúmeros estudos mostrando redução da função em modelos de DM tipo 1 avaliada in vivo, invasivamente (estudo hemodinâmico) ou não (ecocardiograma), e in vitro (preparação de coração isolado e de músculo isolado) $(25,26)$. No DM tipo 2 as conclusões sobre a função sistólica não são consistentes. Os autores têm relatado desempenho sistólico comprometido em preparação de coração isolado; no entanto, ao estudo ecocardio- gráfico a função não tem sido diferente do normal (27). A discrepância entre os resultados dos vários estudos parece estar relacionada às diferentes condições experimentais e à disponibilidade de substratos nos meios de perfusão (23).

Recentemente demonstramos redução dos índices de encurtamento da parede do VE avaliados por ecocardiograma em ratos com DM induzido por estreptozotocina. Nesse experimento, no estudo da função miocárdica em preparações com músculo papilar do VE, observamos lentificação da contração e do relaxamento miocárdico. Além da hiperglicemia, houve aumento dos níveis séricos de colesterol total, LDL-colesterol e triglicérides e diminuição do HDLcolesterol. A atividade do estresse oxidativo avaliada pelos níveis séricos de hidroperóxido e lipoperóxido estava elevada, e foi interessante observar que a administração do antioxidante rutina reverteu, total ou parcialmente, as alterações bioquímicas séricas e funcionais do coração $(24,28)$.

\section{FISIOPATOLOGIA}

A miocardiopatia diabética é vista, atualmente, como resultado de complexas relações entre anormalidades metabólicas que acompanham o diabetes e suas conseqüências celulares, levando à alteração da estrutura e função miocárdica (23). Os três distúrbios metabólicos característicos do diabetes são: hiperlipidemia (geralmente na forma de aumento de triglicérides e de ácidos graxos livres), hiperinsulinemia nas fases mais precoces e, após falência das células beta-pancreáticas, hiperglicemia. O aumento sérico de lípides, insulina e glicose induz alterações na ativação de fatores de transcrição celular dos miócitos cardíacos que resultam em modificações na expressão gênica e na utilização miocárdica de substratos, crescimento miocárdico, disfunção endotelial e aumento da rigidez miocárdica (29).

$\mathrm{O}$ entendimento dos efeitos celulares causados por estes distúrbios metabólicos nos cardiomiócitos poderia ser útil para prever as alterações estruturais e funcionais que podem ocorrer no coração de pacientes com diabetes. A seguir, apresentaremos evidências experimentais a respeito do papel das alterações metabólicas acima mencionadas sobre o desenvolvimento da miocardiopatia diabética.

\section{Aumento dos ácidos graxos}

Em condições fisiológicas, a glicose é o principal carboidrato utilizado pelo coração. Quando comparado à glicose, os ácidos graxos são os substratos preferidos 
pelas células cardíacas e correspondem a cerca de $70 \%$ do ATP gerado aerobicamente pelo coração (30). A alteração predominante que ocorre no metabolismo cardíaco no DM é a supressão da utilização de glicose e a utilização excessiva de ácidos graxos associada ao estoque intracelular de lípides.

O aumento dos ácidos graxos livres tem papel crítico no desenvolvimento de resistência celular à insulina e hiperinsulinemia compensatória e na disfunção contrátil do miocárdio. Os ácidos graxos livres alteram a transdução do sinal mediado pela insulina tanto por ação na membrana celular, ativando a proteína quinase $\delta$, como por efeitos intracelulares decorrentes do aumento de sua concentração no interior do miócito (31). No DM há aumento da oxidação de ácidos graxos e acúmulo mitocondrial de acil carnitina, levando à piora da fosforilação oxidativa (30). O aumento intracelular dos ácidos graxos livres pode também alterar diretamente a contratilidade miocárdica por meio de encurtamento do potencial de ação e alteração no trânsito intracelular de cálcio. No miócito, as enzimas que catalizam a glicólise são localizadas próximas ao sarcolema e ao retículo sarcoplasmático. O ATP gerado pela glicólise é preferencialmente utilizado por enzimas transportadoras de íons, como a SERCA-2a (enzima responsável pela captação de cálcio pelo retículo sarcoplasmático) e a $\mathrm{Na}^{+}-\mathrm{K}^{+}$ATPase. Assim, a inibição da glicólise cardíaca decorrente do aumento da oxidação de ácidos graxos no diabetes pode alterar o funcionamento dessas enzimas e, conseqüentemente, o trânsito intracelular de cálcio (30). É importante salientar que alterações no trânsito intracelular de cálcio podem também ocorrer por outros mecanismos como, por exemplo, a diminuição na expressão gênica das enzimas SERCA-2a e trocador $\mathrm{Na}^{+} / \mathrm{Ca}^{2+}$ (responsável pela troca $\mathrm{Na}^{+} / \mathrm{Ca}^{2+}$ no sarcole$\mathrm{ma})$, observada em animais com ambos os tipos de diabetes $(32,33)$. Finalmente, o acúmulo de ácidos graxos livres no interior do miócito pode induzir lipotoxicidade e contribuir diretamente para a morte celular por apoptose nas situações em que os ácidos graxos acumulados não sofrem beta-oxidação $(23,29,30,34)$. Os mecanismos responsáveis pela lipotoxicidade ainda não estão completamente definidos. Tem sido aventado que a maior oxidação de ácidos graxos causa aumento das espécies reativas de oxigênio, que podem ocasionar dano celular e induzir apoptose (30).

A partir de estudos experimentais com animais transgênicos, foi demonstrado que o aumento de ácidos graxos circulantes contribui fundamentalmente para o desenvolvimento de resistência à insulina e hiperinsulinemia compensatória, e também afeta diretamente a função cardíaca (23).

\section{Hiperinsulinemia}

Embora as ações celulares da insulina no organismo sejam atenuadas em situações de resistência à insulina, a hiperinsulinemia sistêmica pode acentuar a ação da insulina nos tecidos responsíveis à insulina como o miocárdio, que não manifesta resistência celular à insulina (23).

A hiperinsulinemia tem papel importante em estimular a hipertrofia cardíaca. Atualmente, são reconhecidos pelo menos três mecanismos celulares pelos quais a insulina induz hipertrofia $(23,35)$. Agudamente, a insulina estimula o crescimento do miócito por meio da mesma via pela qual ela media a captação de glicose, a via PI3K $\alpha$ (fosfatidil inositol 3 quinase-a) e em seqüência a Akt-l. A Akt-l fosforila e inativa a GSK-3 $\beta$ (glicogênio sintase quinase- $3 \beta$ ), que é inibidora da transcrição nuclear e governa o processo hipertrófico via NFAT-3 (nuclear factor in activated lymphocytes). Além disso, a Akt-1 ativa a mTOR (mammalian target of rapamycin) com subseqüente ativação da p70-ribossomal S6 quinase-1 (p70rsk), levando a aumento da síntese protéica. Essas ações mitogênicas mediadas pelo receptor da insulina podem ser atenuadas quando a sinalização da insulina pela via PI3K $\alpha /$ Akt-1 é piorada durante hiperinsulinemia crônica (23). Entretanto, a hiperinsulinemia crônica pode aumentar a ativação miocárdica da via Akt- 1 também indiretamente, por meio de aumento da ativação do sistema nervoso simpático mediada pelos receptores $\beta_{2}$-adrenérgicos via proteína quinase $\mathrm{A}$ e $\mathrm{Ca}^{2+}$-calmodulina quinase (36). Esses mecanismos podem predominar quando a sinalização da insulina é atenuada pela via PI3K $\alpha$. Finalmente, outras vias mediadas por insulina e relacionadas ao desenvolvimento de hipertrofia podem estar operativas, principalmente as vias ERK/MAP (extracelular signal-regulated kinase/mitogen-activated protein) (37). Estes dados fortemente sugerem que o hiperinsulinismo crônico está associado à hipertrofia cardíaca, e esta pode iniciar-se muito precocemente no diabetes tipo 2 , uma vez que o hiperinsulinismo precede o desenvolvimento da hiperglicemia.

\section{Hiperglicemia}

Além da lipotoxicidade causada pela hiperlipidemia, a hiperglicemia também causa toxicidade celular, conhecida como glicotoxicidade, que contribui para as alterações cardíacas observadas em doentes com diabetes $(30)$.

A hiperglicemia causa aumento da oxidação da glicose e geração mitocondrial de superóxido. $\mathrm{O}$ estresse oxidativo induzido pela hiperglicemia ativa a 
poli(ADP-ribose) polimerase-1 (PARP) como uma enzima reparativa (23). A ativação da PARP regula várias reações celulares como reparo de DNA, expressão gênica e sobrevida celular. Entretanto, a ativação excessiva da PARP pode iniciar vários processos celulares e causar dano celular. A PARP também tem papel na inibição da enzima desidrogenase gliceraldeído fosfato $(\mathrm{GAPDH})$, derivando a glicose de sua via glicolítica para vias bioquímicas alternativas. Estas vias incluem aumento na formação de produtos finais de glicação avançada (AGEs), aumento de hexosamina e ativação da proteína quinase $\mathrm{C}$, que são consideradas mediadoras da lesão celular induzida por hiperglicemia (23). O aumento de AGEs promove a formação de ligações irreversíveis com várias macromoléculas. Por exemplo, a sua ligação ao colágeno induz fibrose intersticial e a ligação às enzimas SERCA-2a e RyR (canais sensíveis à rianodina, responsáveis pela liberação de cálcio pelo retículo sarcoplasmático) leva à inativação ou redução de suas funções $(30,38,39)$. Além disso, pode haver redução da quantidade de SERCA-2a decorrente de aumento de hexosamina no miócito. Estes dados fornecem evidências para a associação entre hiperglicemia e alteração da expressão e função das enzimas SERCA-2 e RyR, com conseqüente piora do relaxamento, contratilidade e rigidez miocárdica (23). De acordo com a relação entre hiperglicemia e alterações subcelulares, foi verificado que a severidade da disfunção diastólica em diabéticos se correlaciona positivamente com os valores séricos da hemoglobina glicada (40).

Em resumo, levando em conta as alterações cardíacas decorrentes de anormalidades metabólicas em diabéticos, poderia ser possível prever as manifestações da miocardiopatia diabética de acordo com a duração e severidade de alterações na homeostase dos ácidos graxos livres, insulina e glicose (23).

Além das alterações metabólicas, outros fatores também têm sido implicados no desenvolvimento da miocardiopatia diabética. Uma vez iniciadas as alterações subcelulares cardíacas, a ativação de receptores miocitários para estiramento celular leva à ativação do sistema renina-angiotensina-aldosterona e do sistema nervoso simpático. As conseqüências maléficas da ativação excessiva desses sistemas sobre o aparelho cardiovascular já são bem conhecidas, em estudos clínicos e experimentais. Outra alteração que foi observada em corações de ratos diabéticos é a indução do programa de genes fetais, habitualmente encontrada em situações de hipertrofia e insuficiência cardíaca (29). Finalmente, fatores extra-cardíacos como disfunção endotelial, aumento da rigidez arterial (41) e neuropatia autonômica (42) certamente têm influência sobre o comportamento mecânico do coração e, portanto, desempenham papel importante nas manifestações clínicas decorrentes da miocardiopatia diabética.

\section{QUADRO CLÍNICO, DIAGNÓSTICO E TRATAMENTO}

O diagnóstico definitivo de miocardiopatia diabética é difícil de ser estabelecido, principalmente porque os sinais, sintomas e achados de exames diagnósticos são inespecíficos. Além disso, o quadro clínico e laboratorial que levou a suspeita de miocardiopatia diabética pode ser decorrente de co-morbidades muito prevalentes entre os diabéticos, como hipertensão arterial sistêmica, doença aterosclerótica coronariana e obesidade. Mesmo na ausência de co-morbidades, os achados clínicos podem ser indistinguíveis daqueles causados por outras formas de cardiomiopatia. Portanto, na avaliação dos pacientes com hipótese diagnóstica de miocardiopatia diabética, devem ser investigadas outras possíveis causas de comprometimento miocárdico.

Interessantemente, apesar de existirem diferenças entre os modelos experimentais de diabetes tipo l e 2, em humanos as manifestações clínicas são semelhantes nos dois tipos de diabetes. Isso se deve, provavelmente, ao fato de que humanos com diabetes tipo 1 são tratados com insulina e, portanto, não apresentam hipoinsulinemia (23).

Habitualmente, a manifestação clínica da miocardiopatia diabética caracteriza-se por dispnéia devido à congestão pulmonar decorrente da disfunção diastólica do VE. Posteriormente, com o avanço da doença, pode ocorrer comprometimento do desempenho sistólico agravando o quadro de IC. Os sinais e sintomas de IC direita, assim como a forma clínica de miocardiopatia dilatada com IC global, não são comuns na miocardiopatia diabética (43).

Além da IC, pacientes com DM, quando comparados aos não-diabéticos, apresentam maior incidência de arritmias, principalmente extra-sístoles ventriculares e supraventriculares e até mesmo fibrilação ventricular e morte súbita (44). Evidências sugerem que os distúrbios de ritmo e de condução podem ser causados por neuropatia autonômica, hipertrofia miocárdica e fibrose intersticial observadas na miocardiopatia diabética.

O ecocardiograma é o exame mais indicado, considerando custo e benefício, na avaliação estrutural e funcional do coração de pacientes com DM. A hipertrofia do VE pode ser observada em até um terço dos pacientes com DM tipo 2, independentemente 
dos valores da pressão arterial ou do uso de inibidores da enzima conversora de angiotensina (45). Como referido anteriormente, acredita-se que a hipertrofia seja causada por uma combinação de fatores como hiperinsulinemia, ativação dos sistemas neuro-hormonais renina-angiotensina-aldosterona e sistema nervoso simpático, e também pelo aumento da rigidez aórtica com conseqüente elevação do estresse sistólico na parede do VE. Apesar de evidências de que a hipertrofia seria um dos principais fatores causadores de disfunção diastólica do VE, ainda não está definido se as anormalidades da função diastólica são decorrentes da hipertrofia ou hiperglicemia (23). Com a complementação do exame ecocardiográfico convencional com a técnica do Doppler tissular foi possível identificar disfunção diastólica do VE com fração de ejeção normal em até $75 \%$ dos pacientes diabéticos, normotensos, não coronarianos e sem sinais ou sintomas de IC (20). Apesar da fração de ejeção normal e da ausência de IC, há estudos mostrando ser possível a detecção de algum grau de disfunção sistólica pelo Doppler tissular $(22,46)$.

A dosagem do peptídeo natriurético cerebral (BNP) realizada em pacientes diabéticos sem IC não se mostrou útil na identificação de portadores de disfunção diastólica observada ao ecocardiograma (47).

No tratamento da miocardiopatia diabética é de fundamental importância o controle do DM de acordo com as diretrizes vigentes, o controle do peso corporal, a alimentação saudável e a atividade física regular, além do controle rigoroso de doenças associadas, principalmente hipertensão arterial, doença coronariana e dislipidemia.

O tratamento da IC e da arritmia por miocardiopatia diabética não difere do tratamento de miocardiopatias de outras etiologias (48). A seguir serão descritas algumas particularidades do tratamento da IC em pacientes com DM.

\section{Betabloqueadores}

Ainda hoje existe alguma resistência para o uso de betabloqueadores em pacientes diabéticos devido ao seu efeito desfavorável à resistência insulínica ou à dificuldade de percepção da hipoglicemia por parte do paciente. No entanto, com a melhor compreensão da IC e do papel deletério da hiperatividade do sistema adrenérgico no estado hemodinâmico e na remodelação cardíaca, os betabloqueadores mostraram-se essenciais no tratamento da IC. Alguns estudos sugerem que, em relação aos demais betabloqueadores, o carvedilol teria vantagens em pacientes diabéticos com IC devido ao seu efeito favorável sobre a sensibi- lidade à insulina e os lipídeos séricos, assim como a sua atividade vasodilatadora periférica. Recomenda-se não utilizar betabloqueadores com atividade simpatomimética intrínseca, como pindolol, particularmente em pacientes diabéticos com IC.

\section{Inibidores da enzima conversora de angiotensina (IECA)}

Os inúmeros ensaios clínicos com o uso de IECA mostraram que esse grupo de fármacos é imprescindível no tratamento da IC devido ao efeito benéfico na redução de morbidade e mortalidade. Além disso, foi demonstrado que o efeito favorável é ainda maior nos pacientes diabéticos.

\section{Bloqueadores do receptor da angiotensina II (BRA)}

Em pacientes diabéticos, o benefício desse grupo de drogas parece ser inferior aos IECA no tratamento da IC. Portanto, a recomendação atual é utilizar esses bloqueadores somente em pacientes intolerantes aos IECA. Importante ressaltar que os grandes benefícios dos medicamentos na morbidade e mortalidade foram demonstrados em pacientes que apresentavam comprometimento da função sistólica do VE. Nos poucos ensaios clínicos que incluíram pacientes com IC e fração de ejeção normal do VE e, portanto, supostamente portadores de disfunção diastólica isolada, os resultados foram inconclusivos. Como na grande maioria dos pacientes com miocardiopatia diabética, principalmente na fase inicial, a IC é decorrente de disfunção diastólica isolada, habitualmente o tratamento consiste em reduzir os sintomas de congestão pulmonar e/ou periférica (diuréticos), controlar a freqüência ventricular em doentes com fibrilação atrial (betabloqueadores, digital ou antagonistas de cálcio) e tratar rigorosamente as co-morbidades como hipertensão arterial sistêmica e doença coronariana (betabloqueadores, IECA, BRA ou antagonistas de cálcio).

\section{REFERÊNCIAS}

1. Wild S, Roglic G, Green A, Sicree R, King H. Global prevalence of diabetics: estimates for the year 2000 and projections for 2030. Diabetes Care 2004;27:1047-53.

2. Malerbi DA, Franco LJ. Multicenter study of the prevalence of diabetes mellitus and impaired glucose tolerance in the urban Brazilian population aged 30-69 yr. The Brazilian Cooperative Group on the Study of Diabetes Prevalence. Diabetes Care 1992;15:1509-16.

3. Torquato MT, Montenegro RM Jr, Viana LA, Souza RA, Lanna $\mathrm{CM}$, Lucas JC, et al. Prevalence of diabetes mellitus and impaired glucose tolerance in the urban population aged 30-69 years in Ribeirão Preto (São Paulo), Brazil. São Paulo Med J 2003; 121:224-30. 
4. Kannel WB, Hjortland M, Castelli WP. Role of diabetes in congestive heart failure: the Framingham study. Am J Cardiol 1974;34:29-34.

5. Giugliano D, Ceriello A, Paoglisso G. Oxidative stress and diabetic vascular complications. Diabetes Care 1996;19:25765.

6. Gu K, Cowie CC, Harris ML. Diabetes and decline in heart disease mortality in U.S. adults. JAMA 1999;281:1291-7.

7. Rubler S, Dluglash J, Yuceoglu YZ, Kumral T, Branwood AW, Grishman A. New type of cardiomyopathy associated with diabetic glomerulosclerosis. Am J Cardiol 1972;30:595602.

8. Codinach-Huix P, Freixa-Pamias R. Diabetic cardiomyopathy: concept, heart function, and pathogenesis. An Med Intern 2002; 19:313-20.

9. Kannel WB, Hjortland M, Castelli WP. Role of diabetes in congestive heart failure. Am J Cardiol 1974;34:29-34.

10. Shindler DM, Kostis JB, Yusuf S, Quinones MA, Pitt B, Stewart $D$, et al. Diabetes mellitus, a predictor of morbidity and mortality in the Studies of the Left Ventricular Dysfunction (SOLVD) Trials and Registry. Am J Cardiol 1996;77:1017-20.

11. Ryden L, Armstrong PW, Cleland JG, Horowitz JD, Massie BM, Packer M, et al. Efficacy and safety of high-dose lisinopril in chronic heart failure patients at high cardiovascular risk, including those with diabetes mellitus. Results from the ATLAS trial. Eur Heart J 2000;21:1967-78.

12. Cohn JN, Johnson G, Ziesche S, Cobb F, Francis G, Tristani F, et al. A comparison of enalapril with hydralazine-sosorbide dinitrate in the treatment of chronic congestive heart failure. N Engl J Med 1991;325:303-10.

13. Rossen JD. Abnormal microvascular function in diabetes: relationship to diabetic cardiomyopathy. Coron Artery Dis 1996;7:133-8.

14. Zoneraich S, Silverman G, Zoneraich O. Primary myocardial disease, diabetes mellitus, and small vessel disease. Am Heart J 1980;100:754-5.

15. Gutierrez PS, Higuchi ML. Alterações cardíacas e vasculares no diabetes: aspectos anatomopatológicos. Rev Soc Cardiol Estado São Paulo 1998;8:1020-4.

16. Chaves FR, Jorge PAR. Miocardiopatia diabética. Arq Bras Endocrinol Metab 1998;42:134-9.

17. Stratton IM, Adler AI, Neil HA, Matthews DR, Manley SE, Cull $\mathrm{CA}$, et al. Association of glycaemia with macrovascular and microvascular complications of type 2 diabetes (UKPDS 35): prospective observational study. BMJ 2000;321:405-12.

18. Adler AI, Stratton IM, Neil HA, Yudkin JS, Matthews DR, Cull CA, et al. Association of systolic blood pressure with macrovascular and microvascular complications of type 2 diabetes (UKPDS 36): prospective observational study. BMJ 2000;321:412-9.

19. Mukamal KJ, Nesto RW, Cohen MC, Muller JE, Maclure M, Sherwood JB. Impact of diabetes on long-term survival after acute myocardial infarction: comparability of risk with prior myocardial infarction. Diabetes Care 2001;24:1422-7.

20. Boyer JK, Thanigaraj S, Schechtman KB, Perez JE. Prevalence of ventricular diastolic dysfunction in asymptomatic, normotensive patients with diabetes mellitus. Am J Cardiol 2004:93:870-5.

21. Braga JC, Guimarães Filho FV, Padovani CR, Matsubara BB. Diastolic dysfunction in diabetic normotensive patients, regardless of the presence of microangiopathy. Arq Bras Cardiol 2005;84:461-6.

22. Gonzalez-Vilchez F, Ayuela J, Ares M, Pi J, Catillo L, MartinDuran R. Oxidative stress and fibrosis in incipient myocardial dysfunction in type 2 diabetic patients. Int $J$ Cardiol 2005;101:53-8.

23. Poornima IG, Parikh P, Shannon RP. Diabetic cardiomyopathy: the search for a unifying hypothesis. Circ Res 2006;98:596-605.

24. Di Muzio BP, Okoshi K, Cicogna AC, Novelli ELB, Dal Pai Silva M, Okoshi MP, et al. Avaliação funcional (in vivo e in vitro) e histológica do ventrículo esquerdo e bioquímica de ratos com diabetes mellitus induzido por estreptozotocina. Rev Soc Cardiol Estado de São Paulo 2006;16:144.
25. Joffe II, Travers KE, Perreault-Micale CL, Hampton T, Katz SE, Morgan JP, et al. Abnormal cardiac function in the streptozotocin-induced, non-insulin-dependent diabetic rat. J Am Coll Cardiol 1999;34:2111-9.

26. Trost SU, Belke DD, Bluhm WF, Meyer M, Swanson E, Dillmann WH. Overexpression of the sarcoplasmic reticulum $\mathrm{Ca}(2+)-A T P a s e$ improves myocardial contractility in diabetic cardiomyopathy. Diabetes 2002;51:1166-71.

27. Wang P, Lloyd SG, Zeng H, Bonen A, Chatham JC. Impact of altered substrate utilization on cardiac function in isolated hearts from Zucker diabetic fatty rats. Am J Physiol Heart Circ Physiol 2005;288:H2102-10.

28. Guimarães JFC, Okoshi K, Cicogna AC, Padovani CR, Cal Pai Silva M, Okoshi MP, et al. Avaliação funcional (in vivo e in vitro) e histológica do ventrículo esquerdo e bioquímica de ratos diabéticos tratados com o flavonóide rutina. Rev Soc Cardiol Estado de São Paulo 2006;16:36.

29. Hayat SA, Patel B, Khattar RS, Malik RA. Diabetic cardiomyopathy: mechanisms, diagnosis and treatment. Clin Sci 2004; 107:539-57.

30. Ding A, Rodrigues $B$. Role of changes in cardiac metabolism in development of diabetic cardiomyopathy. Am J Physiol Heart Circ Physiol 2006;291:H1489-506.

31. Kim JK, Kim YJ, Fillmore JJ, Chen Y, Moore I, Lee J, et al. Prevention of fat-induced insulin resistance by salicylate. $\mathbf{J} \mathbf{C l i n}$ Invest $2001 ; 108: 437-46$

32. Hattori Y, Matsuda N, Kimura J, Ishitani T, Tamada A, Gando $S$, et al. Diminished function and expression of the cardiac $\mathrm{Na}^{+}-\mathrm{Ca}^{2}$ exchanger in diabetic rats: implication in $\mathrm{Ca}^{2}$ overload. J Physiol 2000;527:85-94.

33. Belke DD, Swanson EA, Dillmann WH. Decreased sarcoplasmic reticulum activity and contractility in diabetic $\mathrm{db} / \mathrm{db}$ mouse heart. Diabetes 2004;53:3201-8.

34. Zhou YT, Grayburn P, Karim A, Shimabukuro M, Higa M, Baetens D, et al. Lipotoxic heart disease in obese rats: implications for human obesity. Proc Natl Acad Sci USA 2000;97:1784-9.

35. Ueno M, Carvalheira JB, Tambascia RC, Bezerra RM, Amaral ME, Carneiro EM, et al. Regulation of insulin signalling by hyperinsulinaemia: role of IRS-1/2 serine phosphorylation and the mTOR/p70 S6K pathway. Diabetologia 2005;4:50618.

36. Kern W, Peters A, Born J, Fehm HL, Schultes B. Changes in blood pressure and plasma catecholamine levels during prolonged hyperinsulinemia. Metabolism 2005;54:391-6.

37. Wang CC, Goalstone ML, Draznin B. Molecular mechanisms of insulin resistance that impact cardiovascular biology. Diabetes 2004:53:2735-40.

38. Bidasee KR, Nallani K, Yu Y, Cocklin RR, Zhang Y, Wang M, et al. Chronic diabetes increases advanced glycation end products on cardiac ryanodine receptors/calcium-release channels. Diabetes 2003;52:1825-36.

39. Bidasee KR, Zhang Y, Shao $\mathrm{CH}$, Wang M, Patel KP, Dincer UD, et al. Diabetes increases formation of advanced glycation end products on Sarco(endo)plasmic reticulum $\mathrm{Ca}^{2+-A T P a s e}$. Diabetes 2004; $53: 463-73$.

40. Iribarren C, Karter AJ, Go AS, Ferrara A, Liu JY, Sidney S, et al. Glycaemic control and heart failure among adult patients with diabetes. Circulation 2001;103:2668-73.

41. Matsubara BB, Okoshi K, Okoshi MP, Matsubara LS, Pimenta WP, Christovan JC, et al. Disfunção arterial como possível mecanismo de agressão cardíaca em pacientes com diabetes mellitus tipo II. Estudo doppler-ecocardiográfico. Arq Bras Cardiol 1997;69:155-9.

42. Di Carli MF, Bianco-Batlles D, Landa ME, Kazmers A, Groehn $\mathrm{H}$, Muzik O, et al. Effects of autonomic neuropathy on coronary blood flow in patients with diabetes mellitus. Circulation 1999;100:813-9.

43. Buyukgebiz A, Saylam G, Dundar B, Bober E, Unal N, Akcoral A. Dilated cardiomyopathy as the first early complication in a 14 year-old girl with diabetes mellitus type 1. J Pediatr Endocrinol Metab 2000;13:1143-6. 
44. Schannwell CM, Schneppenheim M, Pering S, Plehn G, Strauer BE. Left ventricular diastolic dysfunction as an early manifestation of diabetic cardiomyopathy. Cardiology 2002;98:33-9.

45. Struthers $A D$, Morris $A D$. Screening for and treating left ventricular abnormalities in diabetes mellitus: a new way of reducing cardiac deaths. Lancet 2002;359:1430-2.

46. Von Bibra H, Thrainsdottir IS, Hansen A, Dounis V, Malmberg $\mathrm{K}$, Ryden L. Tissue Doppler imaging for the detection and quantitation of myocardial dysfunction in patients with type 2 diabetes mellitus. Diab Vasc Dis Res 2005;2:24-30.

47. Valle R, Bagolin E, Canali C, Giovinazzo P, Barro S, Aspromonte N, et al. The BNP assay does not identify mild left ventricular diastolic dysfunction in asymptomatic diabetic patients. Eur J Echocardiogr 2006;7:40-4.
48. Revisão das II Diretrizes da Sociedade Brasileira de Cardiologia para o diagnóstico e tratamento da insuficiência cardíaca. Arq Bras Cardiol 2002;79:1-30.

\section{Endereço para correspondência:}

Katashi Okoshi

Departamento de Clínica Médica

Faculdade de Medicina de Botucatu, Unesp

Distrito de Rubião Júnior, s/n

18618-000 Botucatu, SP

E-mail: katashi@fmb.unesp.br 